\title{
Thyroid blood flow in inferior thyroid artery as predictor for increase in levothyroxine dosage during pregnancy in women with Hashimoto's thyroiditis - a retrospective study
}

Masafumi Kurajoh ${ }^{1 *}$ D, Akiyo Yamasaki ${ }^{1}$, Toshiki Nagasaki ${ }^{1}$, Yuki Nagata ${ }^{1}$, Shinsuke Yamada ${ }^{1}$, Yasuo Imanishi ${ }^{1}$, Masanori Emoto ${ }^{1}$, Kanae Takahashi ${ }^{2}$, Kouji Yamamoto ${ }^{2}$, Ayumi Shintani ${ }^{2}$ and Masaaki Inaba ${ }^{1}$

\begin{abstract}
Background: We examined whether inferior thyroid artery peak systolic velocity (ITA-PSV) predicts an increase in levothyroxine (LT4) dosage in pregnant women with Hashimoto's thyroiditis.

Methods: Twenty-two women with Hashimoto's thyroiditis who were planning and later achieved pregnancy or confirmed as pregnant were enrolled in this retrospective longitudinal observational study. ITA-PSV and thyroid volume were measured using ultrasonography. Serum concentrations of free thyroxine (F-T4), free triiodothyronine (F-T3), and thyroid stimulating hormone (TSH) were simultaneously determined. We adjusted LT4 dosage to maintain serum TSH at $<2.5 \mu \mathrm{IU} / \mathrm{mL}$ (1 st trimester) and later at $<3 \mu \mathrm{IU} / \mathrm{mL}$ (2nd, 3rd trimester).
\end{abstract}

Results: Eighteen patients (81.8\%) required an increase in LT4 dosage during pregnancy, of whom 7 (31.8\%) required an increase $\geq 50 \mu \mathrm{g}$. Multivariable regression analysis showed that TSH ( $\beta=0.507, p=0.008)$ and ITA-PSV ( $\beta=-0.362, p=0.047)$, but not thyroid volume, F-T4, or F-T3, were independently associated with increased LT4 dosage. Receiver-operating characteristic analysis for predicting an increase in LT4 $\geq 50 \mu \mathrm{g} /$ day showed that the area under the curve (0.905) for ITA-PSV with TSH was not significantly increased ( $p=0.123$ ) as compared to that (0.743) for TSH alone, whereas integrated discrimination improvement was significantly increased $(27.9 \%, p=0.009)$.

Conclusions: In pregnant patients with Hashimoto's thyroiditis, ITA-PSV was a significant predictor of increase in LT4 dosage independent of TSH level, while ITA-PSV plus TSH showed significantly improved predictability as compared to TSH alone. These results suggest that ITA-PSV reflects residual thyroid function and is useful for evaluating the need for increased thyroid hormone production in pregnant patients with Hashimoto's thyroiditis.

Keywords: ITA-PSV, Hashimoto's thyroiditis, Pregnancy, Levothyroxine dosage

\footnotetext{
* Correspondence: m1155129@med.osaka-cu.ac.jp

${ }^{1}$ Department of Metabolism, Endocrinology, and Molecular Medicine, Osaka

City University Graduate School of Medicine, 1-4-3, Asahi-machi, Abeno-ku,

Osaka 545-8585, Japan

Full list of author information is available at the end of the article
}

(c) The Author(s). 2019 Open Access This article is distributed under the terms of the Creative Commons Attribution 4.0 International License (http://creativecommons.org/licenses/by/4.0/), which permits unrestricted use, distribution, and reproduction in any medium, provided you give appropriate credit to the original author(s) and the source, provide a link to the Creative Commons license, and indicate if changes were made. The Creative Commons Public Domain Dedication waiver (http://creativecommons.org/publicdomain/zero/1.0/) applies to the data made available in this article, unless otherwise stated. 


\section{Background}

Hypothyroidism during pregnancy, even when subtle, is considered to increase the risk of miscarriage, gestational hypertension, placental abruption, preeclampsia, and postpartum hemorrhage, as well as psychoneurological disorders in the child [1-4]. Therefore, levothyroxine (LT4) replacement is recommended to maintain the serum concentration of thyroid stimulating hormone (TSH) at $<2.5$ $\mu \mathrm{IU} / \mathrm{ml}$ (1st trimester) and later at $<3 \mu \mathrm{IU} / \mathrm{ml}$ (2nd, 3rd trimester) in women with hypothyroidism during pregnancy [5]. The clinical practice guidelines of the Endocrine Society recommend starting LT4 dosage at $50 \mu \mathrm{g} /$ day or more in pregnant women with hypothyroidism if the serum TSH level is in a range of $2.5-10 \mu \mathrm{IU} / \mathrm{ml}$ [6]. However, not all pregnant women with hypothyroidism are required to increase LT4 dosage to maintain the serum level of TSH [4, $7,8]$, and wide inter-individual differences in regard to increased LT4 dosage have been reported [5]. Although serum TSH level prior to pregnancy is known to be a predictor of the requirement for increasing LT4 dosage [9], there are no other known predictors of that in women with primary hypothyroidism.

Importantly, pregnant women with hypothyroidism acquired due to radioablation or surgery require a greater increase in LT4 dosage as compared to those with primary hypothyroidism [10], indicating that thyroid function reserve is closely associated with an increase in LT4 dosage during pregnancy. However, the clinical significance of inferior thyroid artery peak systolic velocity (ITA-PSV) has not been shown in patients with euthyroid Hashimoto's disease, though we previously reported that ITA-PSV reflects thyroid gland capacity to produce thyroid hormone in not only untreated Graves' disease [11] but also euthyroid Graves' disease [12, 13]. Based on these findings, we postulated that ITA-PSV might reflect thyroid function reserve in patients with Hashimoto's thyroiditis. Here, we examined the relationship of ITA-PSV with an increase in LT4 dosage in women with Hashimoto's thyroiditis during pregnancy.

\section{Methods}

\section{Study design and participants}

This was a retrospective longitudinal observational study. Among female patients with Hashimoto's thyroiditis who visited the Outpatient Clinic of the Department of Endocrinology, Osaka City University Hospital, for examination and treatment of that disease from December 2012 to January 2015, those planning pregnancy or at an early stage of pregnancy were initially considered eligible to participate in the present study $(n=59)$. Serum levels of free thyroxine (F-T4), free triiodothyronine (F-T3), and TSH were assayed using an ELECSYS ${ }^{\text {тм }}$ system (Roche Diagnostics K.K., Tokyo, Japan), while ITA-PSV and thyroid volume were assessed by use of an ultrasonography method, as described later. Hashimoto's thyroiditis was diagnosed based on a combination of clinical features, including diffuse swelling of the thyroid gland, presence of anti-thyroid microsomal or antithyroid peroxidase and anti-thyroglobulin antibodies, primary hypothyroidism without another cause to induce hypothyroidism, and appearance of hypoechoic and/or inhomogeneous pattern on thyroid sonogram images [14]. Those who did not become pregnant, transferred to another hospital, or had a miscarriage were excluded $(n=37)$. As a result, 22 women with Hashimoto's thyroiditis who gave birth were retrospectively analyzed in the present study. The subjects were informed verbally regarding the study protocol, and notified that their medical records and charts may be used for research purposes. Additionally, those details as well as the opt-out method were explained in instructions posted on the hospital website (http://www.med.osakacu.ac.jp/interm $2 /$ research/optout.shtml). The study protocol and opt-out option were approved by the Ethics Committee of Osaka City University Graduate School of Medicine (approval No. 4070). Following approval of the study protocol, as well as receipt of administrative permission to access the medical records and charts, all data subjected to analysis were collected from relevant patient medical records and charts, and samples examined were obtained as part of routine care. The present study was conducted in full accordance with the Declaration of Helsinki.

\section{Ultrasound measurements of blood flow in inferior thyroid artery and thyroid volume}

Using a Hitachi HI VISION Ascendus scanner (Hitachi Medical Corporation, Tokyo, Japan), ITA-PSV and thyroid volume were determined with an EUP-L75 probe operating at $18-5 \mathrm{MHz}$ in both color Doppler and pulsed Doppler modes. Thyroid blood flow at the ITA was measured, as we have previously described [11-13]. Measurements were made at the ITA because this artery contributes strongly to thyroid blood flow, and measurement at this position is straightforward and has a coefficient of variation of less than $5.0 \%$. Briefly, the anglecorrection cursor was positioned parallel to the direction of flow. PSV in the right ITA was automatically calculated with the ultrasound apparatus and used as an index of thyroid blood flow. The period of training for performing ITA-PSV measurements is very short, an average of 1 week, and the clinical procedure requires only a few minutes. All measurements were performed by the same examiner (YA), who was blinded to the individual characteristics of the subjects. The intra- and interobserver coefficient of variation values were less than 5.0 and $3.0 \%$, respectively. Thyroid volume was determined by ultrasonography, based on calculation with 
an ellipsoid model (width $\mathrm{x}$ length $\mathrm{x}$ thickness $\times 0.7$, for each lobe) [15].

\section{Treatment of hypothyroidism with oral LT4}

Serum levels of F-T4, F-T3, and TSH were routinely checked every 4 weeks during pregnancy. LT4 dosage were adjusted to maintain serum TSH at $<2.5 \mu \mathrm{IU} / \mathrm{ml}$ (1st trimester) and later at $<3 \mu \mathrm{IU} / \mathrm{ml}$ (2nd, 3rd trimester), according to the guidelines of the American Thyroid Association [5]. The increase in LT4 dosage was calculated as follows: LT4 dosage at birth - LT4 dosage at baseline.

\section{Statistical analysis}

Values are expressed as median (interquartile range) or number (\%). Spearman's correlation coefficient test was performed to determine correlations between continuous variables. Multivariable regression analyses were performed to assess the independent influence of clinical parameters on increase in LT4 dosage. The clinical practice guidelines of the Endocrine Society recommend starting LT4 dosage at $50 \mu \mathrm{g} /$ day or more in pregnant women with hypothyroidism if the serum TSH level is within a range of $2.5-10 \mu \mathrm{IU} / \mathrm{ml}$ [6], thus simple logistic regression analysis was performed to determine the associations of clinical parameters with an increase in LT $4 \geq 50 \mu$ g. In addition, analyses of the area under the curve (AUC) of receiver operating characteristics (ROC) and integrated discrimination improvement (IDI) were performed to compare the accuracy of prediction of TSH as compared to both TSH and ITA-PSV for an increase in LT4 $\geq 50 \mu \mathrm{g}$. Furthermore, a bootstrap optimism-corrected AUC of predictive performance was calculated for internal validation. Statistical analyses were performed using the Statistical Package for the Social Sciences software, version 18.0 (PASW Statistics version 18.0) and $R$, version 3.3.4 ( $R$ Foundation for Statistical Computing, Vienna, Austria). All reported $p$ values are 2-tailed and were considered to be statistically significant at a value $<0.05$.

\section{Results}

\section{Clinical characteristics of subjects}

Patient characteristics are shown in Table 1. Of the 22 enrolled, 7 (31.8\%) were being administered LT4 at the beginning of study. The median levels of F-T4, F-T3, and TSH were $1.29 \mathrm{ng} / \mathrm{mL}, 2.97 \mathrm{pg} / \mathrm{mL}$, and $1.71 \mu \mathrm{IU} / \mathrm{ml}$, respectively. Twenty (90.9\%) patients were euthyroid and 2 (9.1\%) were subclinical hypothyroid status, based on elevation of serum TSH to above the normal upper limit along with a normal serum level of F-T4 [16]. The median values for ITA-PSV and thyroid volume were $14.8 \mathrm{~cm} / \mathrm{s}$ and $9.2 \mathrm{ml}$, respectively.
Table 1 Clinical characteristics of patients

\begin{tabular}{ll}
\hline No. & 22 \\
\hline Age, years & $34.5(33-37)$ \\
$\mathrm{BMl}, \mathrm{kg} / \mathrm{m}^{2}$ & $20.9(19.3-22.9)$ \\
$\mathrm{F}-\mathrm{T} 4, \mathrm{ng} / \mathrm{ml}$ & $1.29(1.18-1.39)$ \\
$\mathrm{F}-\mathrm{T} 3, \mathrm{pg} / \mathrm{ml}$ & $2.97(2.63-3.08)$ \\
$\mathrm{TSH}, \mathrm{\mu lU} / \mathrm{ml}$ & $1.71(1.24-3.88)$ \\
Thyroid volume, ml & $9.2(7.3-13.1)$ \\
ITA-PSV, cm/s & $14.8(11.4-17.7)$ \\
Baseline LT4 administration, $\mathrm{n}(\%)$ & $7(31.8)$
\end{tabular}

Values are expressed as median (interquartile range) or number (\%) Abbreviations: BMI Body mass index, F-T4 Free thyroxine, F-T3 Free triiodothyronine, TSH Thyroid stimulating hormone, ITA-PSV Inferior thyroid artery peak systolic velocity, LT4 Levothyroxine

\section{Association of ITA-PSV with increase in LT4 independent of serum TSH level}

During pregnancy, $18(81.8 \%)$ of the patients required an increase in LT4 dosage $(12.5-100 \mu \mathrm{g})$. To determine whether clinical parameters including ITA-PSV had a relationship with increased LT4 dosage independent of serum TSH, multivariable regression analyses were performed (Table 2). In model 1, which simultaneously included serum TSH and ITA-PSV, both TSH and ITA-PSV were significantly and independently associated with increased LT4 dosage. Furthermore, when ITA-PSV was replaced with F-T4, F-T3, presence of LT4 administration, or thyroid volume (model 2-5), TSH alone remained significantly and independently associated with an increase in LT4.

\section{Associations of ITA-PSV and TSH with increase in LT4 $\geq$ $50 \mu \mathrm{g}$}

During pregnancy, 7 (31.8\%) patients required a dosage increase of $\geq 50 \mu \mathrm{g}$. Table 3 shows the results of simple logistic regression analysis used to examine associations between clinical parameters and an increase in LT4 $\geq$ $50 \mu$ g. ITA-PSV showed a significant association, while TSH had a tendency to be associated with that increase. In contrast, age, BMI, serum F-T4 and F-T3 levels, thyroid volume, and presence of baseline LT4 administration were not significantly associated with an increase in LT4 $\geq 50 \mu \mathrm{g}$.

\section{Accuracy of TSH alone compared to that with ITA-PSV to} predict LT4 increase $\geq \mathbf{5 0} \boldsymbol{\mu g}$

Serum TSH levels are routinely measured in clinical settings in patients with Hashimoto's thyroiditis, thus we compared the accuracy of serum TSH alone to that combined with ITA-PSV for prediction of an LT4 dose increase $\geq 50 \mu \mathrm{g}$ during pregnancy using ROC analysis. As shown in Fig. 1, the AUC of ITA-PSV in combination with TSH $[0.905,95 \%$ confidence interval (CI), 0.771- 
Table 2 ITA-PSV and TSH independently associated with increase in LT4

\begin{tabular}{|c|c|c|c|c|c|c|c|c|c|c|}
\hline \multirow[b]{2}{*}{ Variables } & \multicolumn{2}{|l|}{ Model 1} & \multicolumn{2}{|c|}{ Model 2} & \multicolumn{2}{|c|}{ Model 3} & \multicolumn{2}{|c|}{ Model 4} & \multicolumn{2}{|c|}{ Model 5} \\
\hline & $\beta$ & $p$ & $\beta$ & p & $\beta$ & p & $\beta$ & $p$ & $\beta$ & $p$ \\
\hline TSH & 0.507 & 0.008 & 0.652 & 0.001 & 0.647 & 0.001 & 0.646 & 0.002 & 0.526 & 0.010 \\
\hline ITA-PSV & -0.362 & 0.047 & & & & & & & & \\
\hline F-T4 & & & -0.194 & 0.268 & & & & & & \\
\hline F-T3 & & & & & -0.187 & 0.287 & & & & \\
\hline Baseline LT4 administration (yes = 1, no = 0) & & & & & & & 0.100 & 0.573 & & \\
\hline Thyroid volume & & & & & & & & & -0.277 & 0.146 \\
\hline adjusted $r^{2} / p$ & \multicolumn{2}{|c|}{$0.475 / 0.001$} & \multicolumn{2}{|c|}{$0.392 / 0.003$} & \multicolumn{2}{|c|}{$0.389 / 0.004$} & \multicolumn{2}{|c|}{$0.361 / 0.005$} & \multicolumn{2}{|c|}{$0.420 / 0.002$} \\
\hline
\end{tabular}

Standardized partial regression coefficient values ( $\beta$ values) and level of significance are shown

$\mathrm{R}^{2}$ : coefficient of determination

Abbreviations: TSH Thyroid stimulating hormone, ITA-PSV Inferior thyroid artery peak systolic velocity, F-T4 Free thyroxine, F-T3 Free triiodothyronine,

LT4 Levothyroxine

1.000] for predicting an increase in LT4 dosage $\geq 50 \mu \mathrm{g}$ was higher as compared to that of TSH alone $(0.743$, 95\% CI, 0.485-1.000), though the difference was not significant $(p=0.123)$. Bootstrap optimism-corrected AUC values for ITA-PSV with TSH (0.903, 95\%CI, $0.810-1.000)$ as well as TSH alone $(0.745$, 95\%CI, 0.5051.000) were similar to the above findings. Furthermore, IDI $(0.279,95 \% \mathrm{CI}, 0.068-0.489, p=0.009)$ showed a significant level of discrimination improvement when ITA-PSV was added to the model (Fig. 2).

\section{Discussion}

The present findings demonstrated that ITA-PSV is significantly associated with an increase in LT4 dosage during pregnancy independent of serum TSH, while FT4, FT3, baseline LT4 administration, and thyroid volume showed no such association. Furthermore, the combination of ITA-PSV with TSH predicted an LT4 dosage increase $\geq 50 \mu \mathrm{g}$ during pregnancy more precisely than TSH alone in pregnant women with Hashimoto's thyroiditis. Together, our results suggest that ITA-PSV

Table 3 Simple logistic regression analysis of factors associated with increase in $\mathrm{LT} 4 \geq 50 \mu \mathrm{g}$

\begin{tabular}{lll}
\hline & OR $(95 \% \mathrm{Cl})$ & $p$ \\
\hline Age, years & $1.123(0.877-1.437)$ & 0.359 \\
$\mathrm{BMl}, \mathrm{kg} / \mathrm{m}^{2}$ & $0.924(0.636-1.344)$ & 0.680 \\
$\mathrm{~F}-\mathrm{T} 4, \mathrm{ng} / \mathrm{ml}$ & $0.191(0.001-56.858)$ & 0.569 \\
$\mathrm{~F}-\mathrm{T} 3, \mathrm{pg} / \mathrm{ml}$ & $0.387(0.033-4.502)$ & 0.448 \\
$\mathrm{TSH}, \mathrm{Il} / \mathrm{ml}$ & $1.800(0.972-3.336)$ & 0.062 \\
Thyroid volume, ml & $0.812(0.604-1.091)$ & 0.166 \\
ITA-PSV, cm/s & $0.571(0.348-0.935)$ & 0.026 \\
Baseline LT4 administration (yes = 1, no = 0) & $2.062(0.313-13.574)$ & 0.451
\end{tabular}

Abbreviations: OR Odds ratio, $\mathrm{Cl}$ Confidence interval, BMI Body mass index, FT4 Free thyroxine, F-T3 Free triiodothyronine, TSH Thyroid stimulating hormone, ITA-PSV Inferior thyroid artery peak systolic velocity,

LT4 Levothyroxine independent of TSH is useful to reflect thyroid function reserve to increase thyroid hormone production during pregnancy.

Previous investigations have shown that 50 to $85 \%$ of women with hypothyroidism require an increase in LT4 dosage during pregnancy $[4,7,8]$, though there are wide inter-individual differences in regard to such an increase given to compensate for the increased demand for thyroid hormone production while pregnant [5]. Consistent with those reports, $81.8 \%$ of the present pregnant patients required an LT4 dosage increase for maintaining serum TSH level within the target range, with that increase level varying from 12.5 to $100 \mu \mathrm{g}$ in the present study. Abalovich $\mathrm{M}$ et al. reported that a higher TSH level prior to pregnancy is predictive of a requirement for increased LT4 dose during pregnancy in women with primary hypothyroidism, in whom serum TSH prior to pregnancy was $<2.5 \mu \mathrm{IU} / \mathrm{ml}$ [9], suggesting that serum TSH level might reflect the current status of thyroid hormone production even in women with euthyroid Hashimoto's thyroiditis. In agreement with that report, the present study found that a high serum level of TSH was independently associated with an increase in LT4 dose and also had a tendency to be associated with an increase in dosage $\geq 50 \mu \mathrm{g}$. However, pregnant women with hypothyroidism due to undergoing radioablation therapy or a total thyroidectomy were previously reported to require a greater increase in LT4 dosage during pregnancy as compared to those with primary hypothyroidism, even when baseline TSH normalized with LT4 replacement therapy did not differ significantly prior to pregnancy between those two patient groups [10]. Those findings reflected a lack of residual thyroid function to increase hormone production in response to an increased hormone demand during pregnancy in the former group of patients in that study.

Although thyroid blood flow was shown to be increased in patients with clinically overt hypothyroidism 


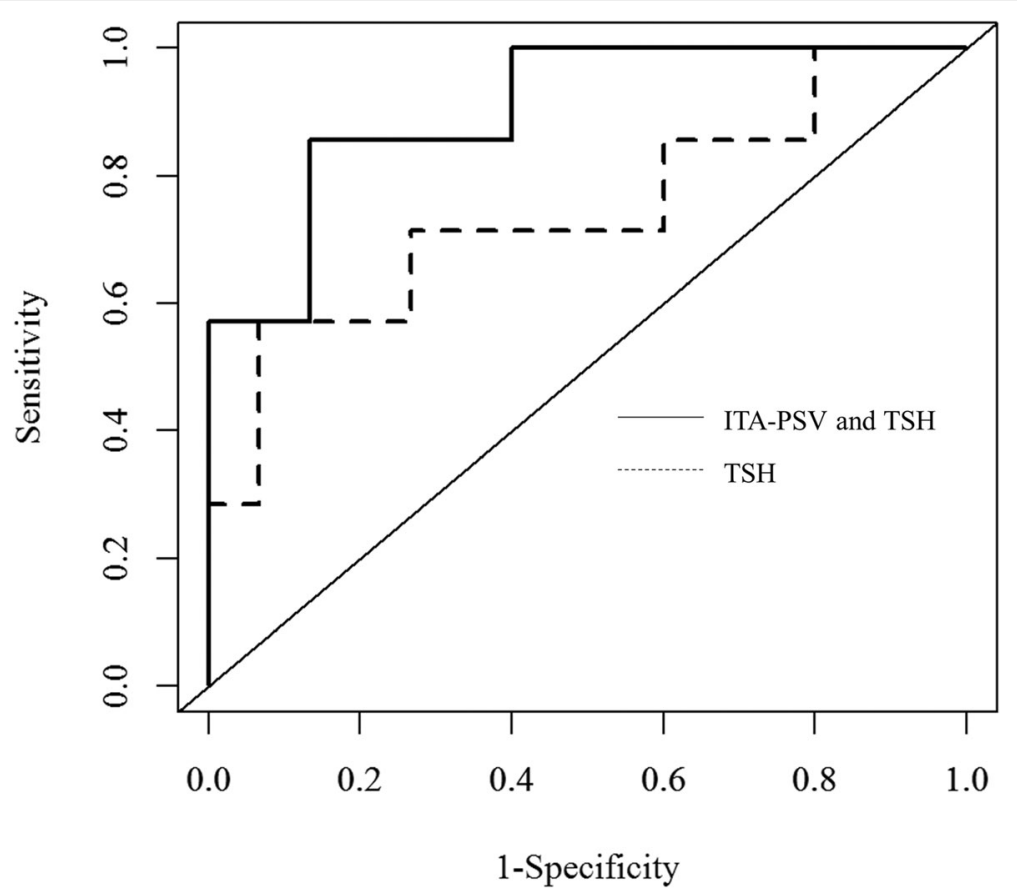

Fig. 1 Receiver operating characteristic analysis of TSH alone as compared to ITA-PSV and TSH to predict increase in LT4 $\geq 50 \mu \mathrm{g}$. The AUC (0.905) value of ITA-PSV and TSH for predicting an increase in LT4 $\geq 50 \mu \mathrm{g}$ was higher as compared to that of TSH alone (0.743), though the difference was not significant $(p=0.123)$. Abbreviations: AUC, area under the curve; TSH, thyroid stimulating hormone; ITA-PSV, inferior thyroid artery peak systolic velocity; LT4, levothyroxine

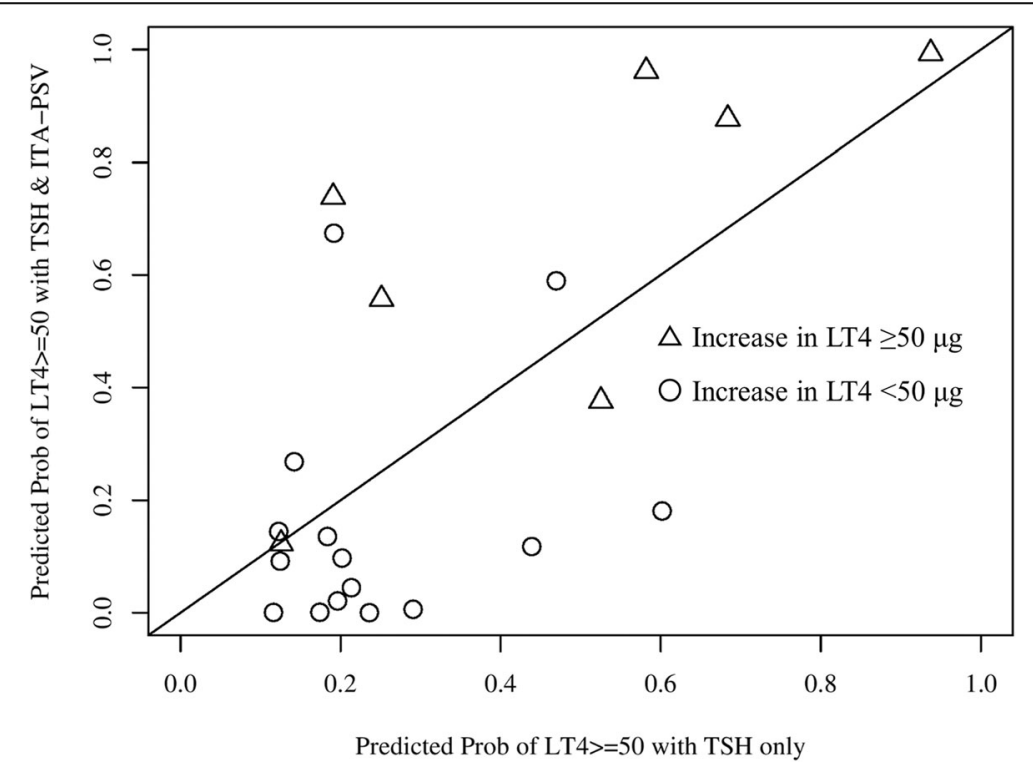

Fig. 2 Integrated discrimination improvement analysis of TSH alone as compared to ITA-PSV and TSH to predict increase in LT4 $\geq 50 \mu g$. The predictability of an increase in LT4 $\geq 50 \mu \mathrm{g}$ based on both ITA-PSV and TSH was significantly increased $(27.9 \%, p=0.009)$ as compared to TSH alone. Abbreviations: TSH, thyroid stimulating hormone; ITA-PSV, inferior thyroid artery peak systolic velocity; LT4, levothyroxine 
as compared to healthy individuals [17], that level was reported to be lower as compared to patients with a TSH-producing tumor [18], suggesting that a rise in ITA-PSV in response to an increase in TSH is weak in patients with Hashimoto's thyroiditis due to destruction of the thyroid gland. Importantly, we previously showed that ITA-PSV predicts Graves' disease relapse after anti-thyroid drug (ATD) withdrawal [12] as well as after normal delivery in patients with euthyroid Graves' disease [13], while a maintenance dosage of ATD is required to keep serum TSH level within a normal range in those patients who are otherwise untreated [11]. Interestingly, in patients with euthyroid Graves' disease, ITA-PSV was shown to be correlated with serum VEGF level, known to be a marker of intra-thyroid angiogenesis, whereas neither serum F-T4 nor F-T3 was found to have a relationship [12], suggesting that ITA-PSV reflects thyroid hormone production in euthyroid Graves' disease. In contrast, we previously reported that ITA-PSV was not correlated with the level of FT4 or F-T3 in healthy subjects [11]. In addition, though ITA-PSV was not significantly correlated with serum F-T4 $(\rho=0.183, p=0.414)$, F-T3 $(\rho=0.091, p=0.687)$, or TSH ( $\rho=-0.262, p=0.239)$ in the present study, it was independently associated with an increase in LT4 dosage, suggesting that ITA-PSV reflects residual thyroid capability to increase thyroid hormone production, but not current thyroid hormone production capability, in patients with euthyroid or subclinical Hashimoto's thyroiditis.

This study has some important limitations. First, it was retrospective in design. Second, the patient cohort was nearly exclusively Japanese individuals, thus it is unclear whether these findings can be generalized for other ethnic groups. Third, the number of subjects investigated was low. Therefore, the power of the present findings is low, and we were not able to fully estimate the association of gestational age with evaluation or sensitivity of ITA-PSV. Our findings should be confirmed in a large-scale study. Nevertheless, to the best of our knowledge, no previously reported study has performed such an examination of predictors of increased LT4 outside of TSH level.

\section{Conclusions}

In pregnant women with Hashimoto's thyroiditis, ITAPSV and TSH levels were independently associated with a required increase in LT4 dosage, while those together more precisely predicted that requirement.

\section{Abbreviations}

ATD: anti-thyroid drug; AUC: area under the curve; Cl: confidence interval; FT3: free triiodothyronine; F-T4: free thyroxine; IDI: integrated discrimination improvement; ITA-PSV: inferior thyroid artery peak systolic velocity; LT4: levothyroxine; ROC: receiver operating characteristics; TSH: thyroid stimulating hormone

\section{Acknowledgements}

We thank all of the investigators and staff who helped with this project.

\section{Authors' contributions}

MK contributed to data analysis, interpretation, and writing of the manuscript. AY, TN, YN, and SY contributed to study design and acquisition of data. YI and ME contributed to critical review of the manuscript. KT, KY, and AS contributed to data analysis and interpretation. MI contributed to data analysis, interpretation, and writing of the manuscript. All authors have approved the final version of the manuscript.

\section{Funding}

No specific grant from a funding agency in the public, commercial, or notfor-profit sectors was received for this research.

\section{Availability of data and materials}

The datasets used and/or analyzed during the current study are available from the corresponding author on reasonable request.

\section{Ethics approval and consent to participate}

All subjects were informed verbally that their medical records and charts may be used for research purposes, and also regarding the opt-out option to withdraw from participation. All were treated as outpatients and obtained samples were examined as part of routine care. An opt-out option is required by the Ethics Committee of Osaka City University Graduate School of Medicine for all studies. Approval of the opt-out option and study protocol, as well as administrative permission to access the medical records and charts were granted by the Ethics Committee of Osaka City University Graduate School of Medicine (approval No. 4070).

\section{Consent for publication}

Not applicable.

\section{Competing interests}

The authors have no competing interests to declare.

\section{Author details}

${ }^{1}$ Department of Metabolism, Endocrinology, and Molecular Medicine, Osaka City University Graduate School of Medicine, 1-4-3, Asahi-machi, Abeno-ku, Osaka 545-8585, Japan. ${ }^{2}$ Department of Medical Statistics, Osaka City

University Graduate School of Medicine, Osaka, Japan.

Received: 6 September 2018 Accepted: 30 June 2019

Published online: 05 July 2019

\section{References}

1. Casey BM, Dashe JS, Wells CE, Mclntire DD, Byrd W, Leveno KJ, Cunningham FG. Subclinical hypothyroidism and pregnancy outcomes. Obstet Gynecol. 2005;105(2):239-45.

2. Haddow JE, Palomaki GE, Allan WC, Williams JR, Knight GJ, Gagnon J, O'Heir CE, Mitchell ML, Hermos RJ, Waisbren SE, et al. Maternal thyroid deficiency during pregnancy and subsequent neuropsychological development of the child. N Engl J Med. 1999;341(8):549-55.

3. Leung AS, Millar LK, Koonings PP, Montoro M, Mestman JH. Perinatal outcome in hypothyroid pregnancies. Obstet Gynecol. 1993;81(3):349-53.

4. Abalovich M, Gutierrez S, Alcaraz G, Maccallini G, Garcia A, Levalle O. Overt and subclinical hypothyroidism complicating pregnancy. Thyroid. 2002;12(1):63-8

5. Stagnaro-Green A, Abalovich M, Alexander E, Azizi F, Mestman J, Negro R, Nixon A, Pearce EN, Soldin OP, Sullivan S, et al. Guidelines of the American Thyroid Association for the diagnosis and management of thyroid disease during pregnancy and postpartum. Thyroid. 2011;21(10):1081-125.

6. De Groot L, Abalovich M, Alexander EK, Amino N, Barbour L, Cobin RH, Eastman CJ, Lazarus JH, Luton D, Mandel SJ, et al. Management of thyroid dysfunction during pregnancy and postpartum: an Endocrine Society clinical practice quideline. J Clin Endocrinol Metab. 2012;97(8):2543-65.

7. Mandel SJ, Larsen PR, Seely EW, Brent GA. Increased need for thyroxine during pregnancy in women with primary hypothyroidism. N Engl J Med. 1990;323(2):91-6.

8. Alexander EK, Marqusee E, Lawrence J, Jarolim P, Fischer GA, Larsen PR. Timing and magnitude of increases in levothyroxine requirements 
during pregnancy in women with hypothyroidism. N Engl J Med. 2004; 351(3):241-9.

9. Abalovich M, Alcaraz G, Kleiman-Rubinsztein J, Pavlove MM, Cornelio C, Levalle O, Gutierrez S. The relationship of preconception thyrotropin levels to requirements for increasing the levothyroxine dose during pregnancy in women with primary hypothyroidism. Thyroid. 2010;20(10):1175-8.

10. Loh JA, Wartofsky L, Jonklaas J, Burman KD. The magnitude of increased levothyroxine requirements in hypothyroid pregnant women depends upon the etiology of the hypothyroidism. Thyroid. 2009;19(3):269-75.

11. Nagasaki T, Inaba M, Kumeda Y, Fujiwara-Ueda M, Hiura Y, Nishizawa Y. Significance of thyroid blood flow as a predictor of methimazole sensitivity in untreated hyperthyroid patients with Graves' disease. Biomed Pharmacother. 2007;61(8):472-6.

12. Ueda M, Inaba M, Kumeda Y, Nagasaki T, Hiura Y, Tahara H, Onoda N, Ishikawa T, Nishizawa Y. The significance of thyroid blood flow at the inferior thyroid artery as a predictor for early Graves' disease relapse. Clin Endocrinol. 2005;63(6):657-62.

13. Nagasaki T, Inaba M, Fujiwara-Ueda M, Nishio J, Kumeda Y, Hiura Y, Tahara H, Ishimura E, Ishiko O, Nishizawa Y. Thyroid blood flow as a useful predictor of relapse of Graves' disease after normal delivery in patients with Graves' disease. Biomed Pharmacother. 2010;64(2):113-7.

14. Caturegli P, De Remigis A, Rose NR. Hashimoto thyroiditis: clinical and diagnostic criteria. Autoimmun Rev. 2014;13(4-5):391-7.

15. Murakami Y, Takamatsu J, Sakane S, Kuma K, Ohsawa N. Changes in thyroid volume in response to radioactive iodine for Graves' hyperthyroidism correlated with activity of thyroid-stimulating antibody and treatment outcome. J Clin Endocrinol Metab. 1996;81(9):3257-60.

16. Peeters RP. Subclinical hypothyroidism. N Engl J Med. 2017;376(26):2556-65.

17. Vitti P, Rago T, Mazzeo S, Brogioni S, Lampis M, De Liperi A, Bartolozzi C, Pinchera A, Martino E. Thyroid blood flow evaluation by color-flow Doppler sonography distinguishes Graves' disease from Hashimoto's thyroiditis. J Endocrinol Investig. 1995;18(11):857-61.

18. Bogazzi F, Bartalena L, Brogioni S, Burelli A, Manetti L, Tanda ML, Gasperi M, Martino $E$. Thyroid vascularity and blood flow are not dependent on serum thyroid hormone levels: studies in vivo by color flow doppler sonography. Eur J Endocrinol. 1999;140(5):452-6.

\section{Publisher's Note}

Springer Nature remains neutral with regard to jurisdictional claims in published maps and institutional affiliations.

Ready to submit your research? Choose BMC and benefit from:

- fast, convenient online submission

- thorough peer review by experienced researchers in your field

- rapid publication on acceptance

- support for research data, including large and complex data types

- gold Open Access which fosters wider collaboration and increased citations

- maximum visibility for your research: over $100 \mathrm{M}$ website views per year

At $\mathrm{BMC}$, research is always in progress.

Learn more biomedcentral.com/submissions 